\title{
Matter effects at the T2HK and T2HKK experiments
}

\author{
Sushant K. Raut ${ }^{1, *}$ \\ ${ }^{1}$ Center for Theoretical Physics of the Universe, \\ Institute for Basic Science (IBS), Daejeon, 34051, South Korea
}

Determining the neutrino mass hierarchy and measuring the CP-violating phase $\delta_{\mathrm{CP}}$ are two of the main aims in neutrino physics today. The upcoming T2HK (with small matter effects and high statistics) and DUNE (with large matter effects) experiments have been shown to have excellent sensitivity to $\delta_{\mathrm{CP}}$ and the neutrino mass hierarchy, respectively. The recent T2HKK proposal aims to improve the hierarchy sensitivity of T2HK by placing one of the two tanks of the HK detector at a site in Korea, to collect data at $\sim 1100 \mathrm{~km}$ baseline. In light of the fact that DUNE will anyway collect data at $\sim 1300 \mathrm{~km}$, we explore whether it is advantageous to collect additional long-baseline data as proposed with T2HKK, or to enhance the $\delta_{\mathrm{CP}}$-precision with the 'conventional' T2HK by keeping both detector tanks in Japan. We do this by comparing the physics reach of these two options in conjunction with DUNE. We find that DUNE+T2HKK is better at excluding the wrong hierarchy, reaching $\sqrt{\Delta \chi^{2}} \gtrsim 13$ irrespective of the true parameters. While DUNE+T2HK can measure $\delta_{\mathrm{CP}}$ with more precision in some parts of the parameter space, both DUNE+T2HK and DUNE+T2HKK perform equally well near the current best-fit point, giving a $\delta_{\mathrm{CP}}$ uncertainty of around $15^{\circ}$. The T2HKK setup allows us to correlate and constrain the systematic errors between the two detectors collecting data from the same source, which can increase the sensitivity of the experiment by up to $25 \%$. Such a reduction of the systematic errors is crucial for determining the oscillation parameters with greater significance. 


\section{INTRODUCTION}

Over the last several decades, experimental tests have confirmed the predictions of the Standard Model of particle physics to an unprecedented level of precision. The Higgs boson that was discovered at the Large Hadron Collider was the last missing piece of the Standard Model [1, 2]. Various hints have emerged for possible new physics beyond the Standard Model at collider experiments. However, the only confirmed signal of physics beyond the Standard Model comes from neutrino oscillation experiments that point to non-zero neutrino mass, contrary to the Standard Model.

Neutrino oscillations in the standard three-flavour scenario are parametrized by three mixing angles $\theta_{12}, \theta_{13}$ and $\theta_{23}$, two mass-squared differences $\Delta m_{21}^{2}$ and $\Delta m_{31}^{2}$ (where $\Delta m_{i j}^{2}=m_{i}^{2}-m_{j}^{2}$ ) and one CP-violating phase $\delta_{\mathrm{CP}}$. While $\theta_{12}, \theta_{13}, \Delta m_{21}^{2}$ and $\left|\Delta m_{31}^{2}\right|$ have been measured quite accurately by solar, atmospheric, long-baseline and reactor neutrino experiments [3-10], the global fits of world neutrino data [11 13$]$ are inconclusive about the sign of $\left|\Delta m_{31}^{2}\right|$ or neutrino mass hierarchy, the octant of the close-to-maximal mixing angle $\theta_{23}$ and the value of $\delta_{\mathrm{CP}}$, the CP phase* The binary question of whether the neutrino mass hierarchy is normal (NH), i.e. $\Delta m_{31}^{2}>0$ or inverted (IH), i.e. $\Delta m_{31}^{2}<0$ is interesting from the point of view of ruling out possible new physics models that favour one hierarchy or the other [17]. The value of $\theta_{23}$ being exactly $45^{\circ}$ or very close to it raises the possibility of a symmetry in the flavour sector such as $\mu-\tau$ symmetry [18]. Finally, a precise measurement of $\delta_{\mathrm{CP}}$ will allow us to observe CP violation in the leptonic sector which may explain the matter-antimatter asymmetry of our Universe through the mechanism of leptogenesis [19, 20].

The current generation of long-baseline experiments $\mathrm{T} 2 \mathrm{~K}$ [21] and $\mathrm{NO} \nu \mathrm{A}$ [22], atmospheric neutrino experiments Super-Kamiokande (SK) [4] and IceCube [23], and reactor neutrino experiments Double Chooz [8], Daya Bay [9] and RENO [10] are collecting data which will help to measure these three unknown parameters in addition to measuring the other parameters with greater precision. The main hurdle in determining the values of the parameters in nature is the existence of degeneracies in this multi-dimensional parameter space. If nature has chosen favourable combinations of the mass hierarchy and $\delta_{\mathrm{CP}}$ [24], then $\mathrm{T} 2 \mathrm{~K}$ and $\mathrm{NO} \nu \mathrm{A}$ themselves will be able to determine the mass hierarchy 25] irrespective of the possible choice of $\delta_{\mathrm{CP}}$ in such scenarios. Meanwhile, a precise measurement of $\delta_{\mathrm{CP}}$ is strongly correlated with a knowledge of the hierarchy and the value of $\theta_{23}$ [26]. However, if nature has chosen unfavourable combinations of the parameters, then a statistically significant determination of the unknown parameters will require the next generation of experiments. These include the long-baseline experiments T2HK [27] and DUNE [28] and the atmospheric neutrino experiments Hyper-Kamiokande (HK) [29], ICAL@INO [30] and PINGU [31]. All these experiments will take advantage of matter effects from neutrinos propagating through the earth.

The HK proposal envisages augmenting the existing SK detector that has a fiducial mass of 22.5 kilotons with two additional Water Cerenkov detector tanks with fiducial mass 187 kilotons each. This new setup will serve as a detector for both neutrinos from the J-PARC beam in Tokai as well as atmospheric neutrinos. Recently, an idea that involves placing one tank of fiducial mass 187 kilotons at a site in Korea in the path of the T2K beam has been proposed [32]. (This is actually based on a much older proposal and its subsequent studies [33 36].) This proposal, called Tokai to Hyper-Kamiokande and Korea (T2HKK) will allow the observation of neutrinos at the detector in Kamioka as well as neutrinos at a far site in Korea that will have experienced matter effects. The advantage of this multi-detector setup is that it simultaneously increases the amount of data at the T2HK baseline of $295 \mathrm{~km}$ and gives access to data at a longer baseline of around $1100 \mathrm{~km}$, corresponding to the location of the proposed detector in Korea. Since neutrino oscillation probabilities depend strongly on the distance travelled and the matter effects experienced by the neutrinos, the addition of information from two different baselines is very effective in breaking the parameter degeneracies [24, 37-45]. In particular, the shorter baseline (Tokai to HK) setup is more effective at measuring $\delta_{\mathrm{CP}}$ while the longer one (Tokai to Korea) is better at excluding the wrong hierarchy.

Yet another long-baseline experiment that is expected to determine the mass hierarchy is the upcoming DUNE experiment. This experiment, with a neutrino beam traversing $1300 \mathrm{~km}$ from Fermilab to SURF will anyway collect neutrino oscillation data with matter effects. Both HK and DUNE are expected to be deployed in the mid-2020s 29, 46]. This presents the neutrino physics community with two options - (a) DUNE collects data at the longer baseline $(1300 \mathrm{~km})$ while the beam from Tokai is intercepted by a 374 kiloton detector at HK, or (b) DUNE collects data at the longer baseline $(1300 \mathrm{~km})$ while the beam from Tokai is observed at a 187 kiloton detector at HK and another 187 kiloton detector in Korea. In this article, we compare the physics capabilities of these two options in measuring $\delta_{\mathrm{CP}}$ and determining the mass hierarchy i.e. without considering the possible existence of sterile neutrino states or non-standard neutrino interactions. Some studies of non-standard interactions in the context of T2HKK can be found in Refs. [48 50 . All the detectors under consideration in this work are capable of detecting atmospheric neutrinos in addition to beam neutrinos [51 54].

\footnotetext{
* Recent data from the $\mathrm{T} 2 \mathrm{~K}$ and $\mathrm{NO} \nu \mathrm{A}$ experiments hint weakly towards a value of $\delta_{\mathrm{CP}}$ close to $-90^{\circ}$ and normal mass hierarchy [14, 15]. However, more data is required before a statistically significant statement can be made about these parameters 16 .

$\dagger$ A recent discussion on the complementarity between HK and DUNE can be found in Ref. [47].
} 
This will significantly improve the hierarchy sensitivity of the experiments. However, we do not take these data into account in this study.

This article is organized as follows. In Section II we discuss the experimental setups that we have considered in this work. Section III discusses the problem of parameter degeneracies and their removal, at the level of probabilities. We discuss the results of our numerical simulations in Section IV, before concluding in Section V.

\section{EXPERIMENTAL SETUPS}

\section{A. T2HK}

Our simulation of T2HK is based on the description provided in Ref. [32]. (See Ref. [29] for the most recent HK design report.) The beam from Tokai is a $1.3 \mathrm{MW}$ beam running for ten years (2.5 years in neutrino mode and 7.5 years in antineutrino mode). The detector site is located at a distance of $295 \mathrm{~km}$ from the source, $2.5^{\circ}$ off the beam axis. The detector performance is consistent with the description in Ref. [32], and the projected systematic errors are taken from the same reference. We use the notation T2HK1/T2HK2 to denote the setup with one/two tanks at the HK location, corresponding to a fiducial mass of 187/374 kilotons. We do not consider staging effects in the deployment of the two detectors. In addition to this large HK detector, we also include the existing SK detector (22.5 kilotons) whenever we simulate T2HK.

\section{B. T2Kor}

In this study, the name T2Kor refers to the setup consisting of the source at Tokai (the same as for T2HK) and detector in Korea. The description of the detector and systematic errors is taken from Ref. [32]. A number of potential sites for the detector in Korea have been studied in the literature. Their baselines vary from around $1000 \mathrm{~km}$ to 1200 $\mathrm{km}$, and their off-axis angles vary from around $1^{\circ}$ to $2.5^{\circ}$. For the purpose of this study, we consider a generic site at a distance of $1100 \mathrm{~km}$ and off-axis angle of $1.5^{\circ}$. The neutrino flux for this off-axis location is taken from Ref. [55]. The detector mass for this setup is always 187 kilotons.

\section{T2HKK}

This is a combination of one detector tank with mass 187 kilotons at the HK site, and another (assumed to be identical) detector tank at the Korean site. Thus, we have the schematic description

$$
\mathrm{T} 2 \mathrm{HKK} \equiv \mathrm{T} 2 \mathrm{HK} 1+\mathrm{T} 2 \mathrm{Kor}
$$

Unless stated otherwise, we always correlate the systematics between T2HK1 and T2Kor. In our simulations, we do not take into account the delay in the deployment of the second detector.

\section{DUNE}

The DUNE experiment that we have considered uses a neutrino beam from Fermilab in the 1.07 MW - $80 \mathrm{GeV}$ proton beam configuration, running for 3.5 years each in the neutrino and antineutrino modes. The data are collected at a liquid argon TPC with fiducial mass 40 kilotons located at SURF, $1300 \mathrm{~km}$ away from the source. The specifications for this experiment are taken from Ref. [28]. Again, we do not consider staging effects in the deployment of the detector.

\section{PROBABILITY LEVEL DISCUSSION AND PARAMETER DEGENERACIES}

The $\nu_{\mu} \rightarrow \nu_{e}$ oscillation probability $P_{\mu e}$ is well suited to measure the mass hierarchy and $\delta_{\mathrm{CP}}$. In terms of the neutrino energy $E$ and distance travelled $L$, this probability can be expressed up to second order in $s_{13}=\sin \theta_{13}$ and 
$\alpha=\Delta m_{21}^{2} /\left|\Delta m_{31}^{2}\right|$ as $[56$

$$
\begin{aligned}
P_{\mu e} & \approx 4 s_{13}^{2} \sin ^{2} \theta_{23} \frac{\sin ^{2}(1-p h \hat{A}) \Delta}{(1-p h \hat{A})^{2}} \\
& +2 h \alpha s_{13} \sin 2 \theta_{12} \sin 2 \theta_{23} \cos \left(\Delta+p h \delta_{\mathrm{CP}}\right) \frac{\sin (\hat{A} \Delta)}{\hat{A}} \frac{\sin (1-p h \hat{A}) \Delta}{(1-p h \hat{A})},
\end{aligned}
$$

where $\Delta=\left|\Delta m_{31}^{2}\right| L / 4 E$ and $\hat{A}=\left|2 E V / \Delta m_{31}^{2}\right|$ with $V$ denoting the Wolfenstein matter potential. We have introduced the binary variables $p=+1(-1)$ for neutrinos(antineutrinos) and $h=+1(-1)$ for $\mathrm{NH}(\mathrm{IH})$.

In the limit of vacuum oscillations, we have

$$
\begin{aligned}
P_{\mu e}(\hat{A} \rightarrow 0) & \approx 4 s_{13}^{2} \sin ^{2} \theta_{23} \sin ^{2} \Delta \\
& +2 h \alpha s_{13} \sin 2 \theta_{12} \sin 2 \theta_{23} \cos \left(\Delta+p h \delta_{\mathrm{CP}}\right) \Delta \sin \Delta,
\end{aligned}
$$

i.e. the leading order term is independent of the hierarchy. In fact, close to the oscillation maximum $\Delta=\pi / 2$, the second term becomes insensitive to the hierarchy as well. This degeneracy between the two hierarchies can be lifted and hierarchy sensitivity restored by increasing the matter density that the neutrino travels through, i.e. by going to a longer baseline. In the neutrino(antineutrino) mode, the probability is higher for $\mathrm{NH}(\mathrm{IH})$ than for $\mathrm{IH}(\mathrm{NH})$, because of the $(1-p h \hat{A})^{2}$ term in the denominator. Close to the oscillation maximum, the equation for $P_{\mu e}$ becomes

$$
\begin{aligned}
P_{\mu e}(\Delta=\pi / 2) & \approx 4 s_{13}^{2} \sin ^{2} \theta_{23} \frac{\cos ^{2}(\hat{A} \pi / 2)}{(1-p h \hat{A})^{2}} \\
& -2 p \alpha s_{13} \sin 2 \theta_{12} \sin 2 \theta_{23} \sin \delta_{\mathrm{CP}} \frac{\sin (\hat{A} \pi / 2)}{\hat{A}} \frac{\cos (\hat{A} \pi / 2)}{(1-p h \hat{A})} .
\end{aligned}
$$

Although the first term increases the probability for $\mathrm{NH}$ compared to $\mathrm{IH}$, this difference can be offset by the second term if $\delta_{\mathrm{CP}}=+90^{\circ}$. Similarly the reduced IH probability can be compensated if $\delta_{\mathrm{CP}}=-90^{\circ}$. Thus, the combinations $\left\{\mathrm{NH}, \delta_{\mathrm{CP}}=+90^{\circ}\right\}$ and $\left\{\mathrm{IH}, \delta_{\mathrm{CP}}=-90^{\circ}\right\}$ suffer from the hierarchy- $\delta_{\mathrm{CP}}$ degeneracy and are unfavourable for measuring these parameters. Conversely, determining these parameters is easier for the favourable combinations $\left\{\mathrm{NH}, \delta_{\mathrm{CP}}=-90^{\circ}\right\}$ and $\left\{\mathrm{IH}, \delta_{\mathrm{CP}}=+90^{\circ}\right\}[24,25]$.

The $\delta_{\mathrm{CP}}$ dependence of $P_{\mu e}$ is present in the second term of Eq. 1. The measurement of $\delta_{\mathrm{CP}}$ suffers from the hierarchy- $\delta_{\mathrm{CP}}$ degeneracy, as discussed earlier. However when matter effects are negligible, we see in Eq. 2 that a clean measurement of $\delta_{\mathrm{CP}}$ is possible, especially near the oscillation maximum. (There are additional complications in the measurement of $\delta_{\mathrm{CP}}$ because of the uncertainty in the value of $\theta_{23}$ which affects the first term in Eq. [2 [26, 57 60]. The $\delta_{\mathrm{CP}}$-independent first term acts as a background for the measurement of the $\delta_{\mathrm{CP}}$-dependent second term - an effect that gets worse with increasing $\theta_{23}$.) Thus, a measurement of the mass hierarchy is facilitated by large matter effects but the opposite holds for $\delta_{\mathrm{CP}}$ measurement. In the specific context of T2HKK, it must be noted that the peak of the flux lies close to the second oscillation maximum of the T2Kor baseline. Since the variation of probability with $\delta_{\mathrm{CP}}$ given by $\mathrm{d} P_{\mu e} / \mathrm{d} \delta_{\mathrm{CP}}$ is greater at the second oscillation maximum $(\Delta=3 \pi / 2)$ than at the first one $(\Delta=\pi / 2)$, the T2Kor baseline also contributes to the $\delta_{\mathrm{CP}}$ sensitivity of the T2HKK setup.

DUNE with a baseline of $1300 \mathrm{~km}$ will have exceptional sensitivity to the mass hierarchy. T2HK on the other hand, because of its relatively short baseline of $295 \mathrm{~km}$ will be able to measure $\delta_{\mathrm{CP}}$ precisely. If one of the two detector tanks of T2HK is moved to a farther location in Korea, it will improve the hierarchy sensitivity of the T2HKK setup. On the other hand, since DUNE will already collect data with matter effects, will it be preferable to retain both detector tanks at the $\mathrm{HK}$ site to improve $\delta_{\mathrm{CP}}$-sensitivity? In the next section, we compare the sensitivities of the DUNE+T2HK2 and DUNE+T2HKK setups to try and answer this question.

\section{RESULTS}

We now discuss the results of our numerical simulations. The experimental setups descibed in Section III are simulated using the GLoBES package [61, 62] along with its auxiliary files [63, 64]. For a given set of 'true' and 'test' oscillation parameters $\vec{p}_{\text {true }}$ and $\vec{p}_{\text {test }}$, the binned true and test event rates $N_{i}^{\text {true }}$ and $N_{i}^{\text {test }}$ are simulated using GLoBES. The test rates are modified $N_{i}^{\text {test }} \rightarrow N_{i}^{\text {test }}(\xi)$ to take systematic effects into account through the nuisance parameter(s) $\xi$. We then perform a $\chi^{2}$ analysis using the method of pulls [65 67]

$$
\chi^{2}\left(\vec{p}_{\text {true }}, \vec{p}_{\text {test }}\right)=\min _{\xi}\left[\left(\sum_{i \in \text { bins }} \frac{\left(N_{i}^{\text {true }}-N_{i}^{\text {test }}(\xi)\right)^{2}}{N_{i}^{\text {true }}}\right)+\frac{\xi^{2}}{\sigma_{\xi}^{2}}\right],
$$



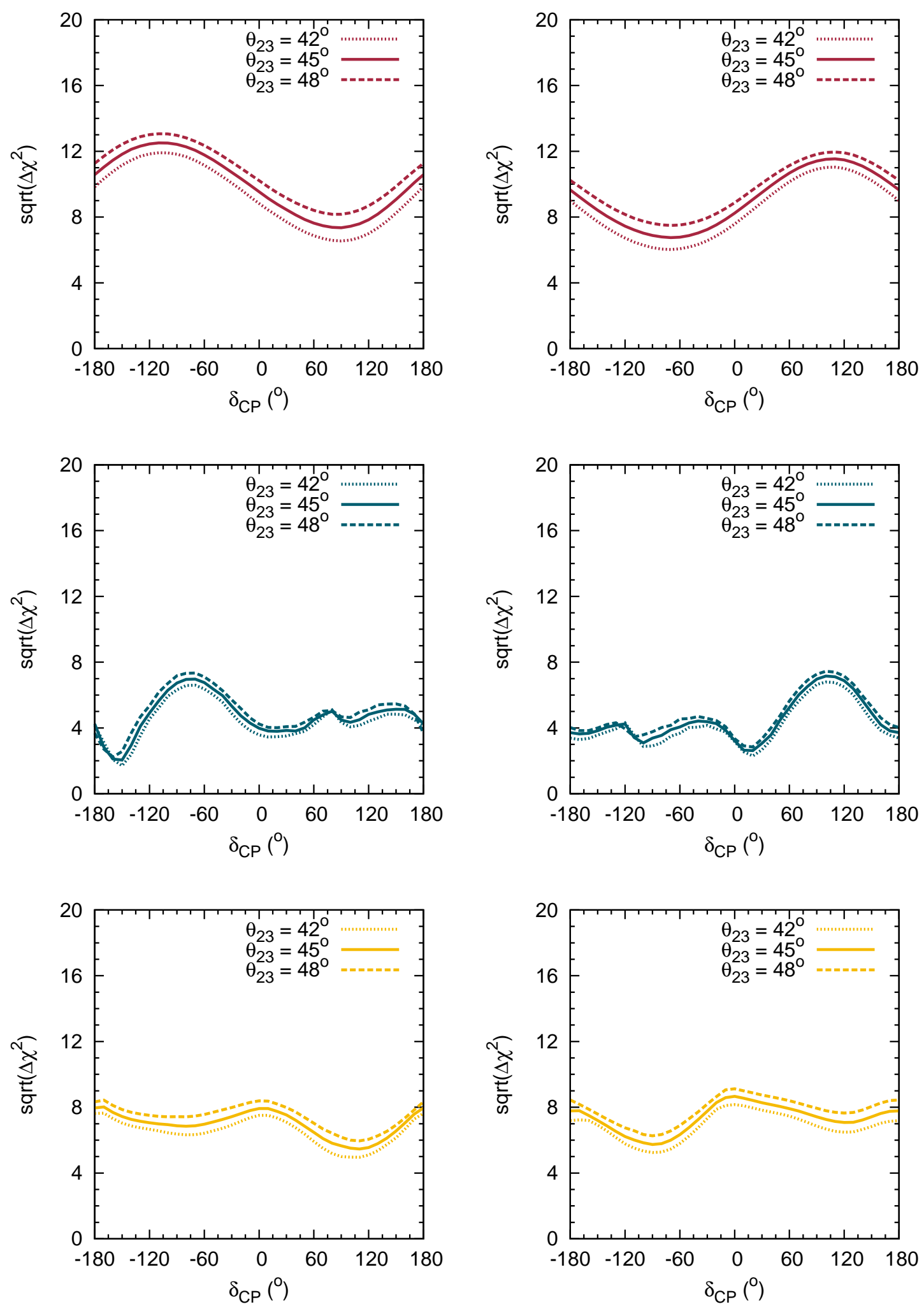

FIG. 1. Hierarchy sensitivity of the experiments DUNE (top row), T2HK2 (middle row) and T2Kor (bottom row) as a function of true $\delta_{\mathrm{CP}}$. The curves correspond to three representative values of $\theta_{23}$ and are shown for both true hierarchies $-\mathrm{NH}$ (left column) and IH (right column). 

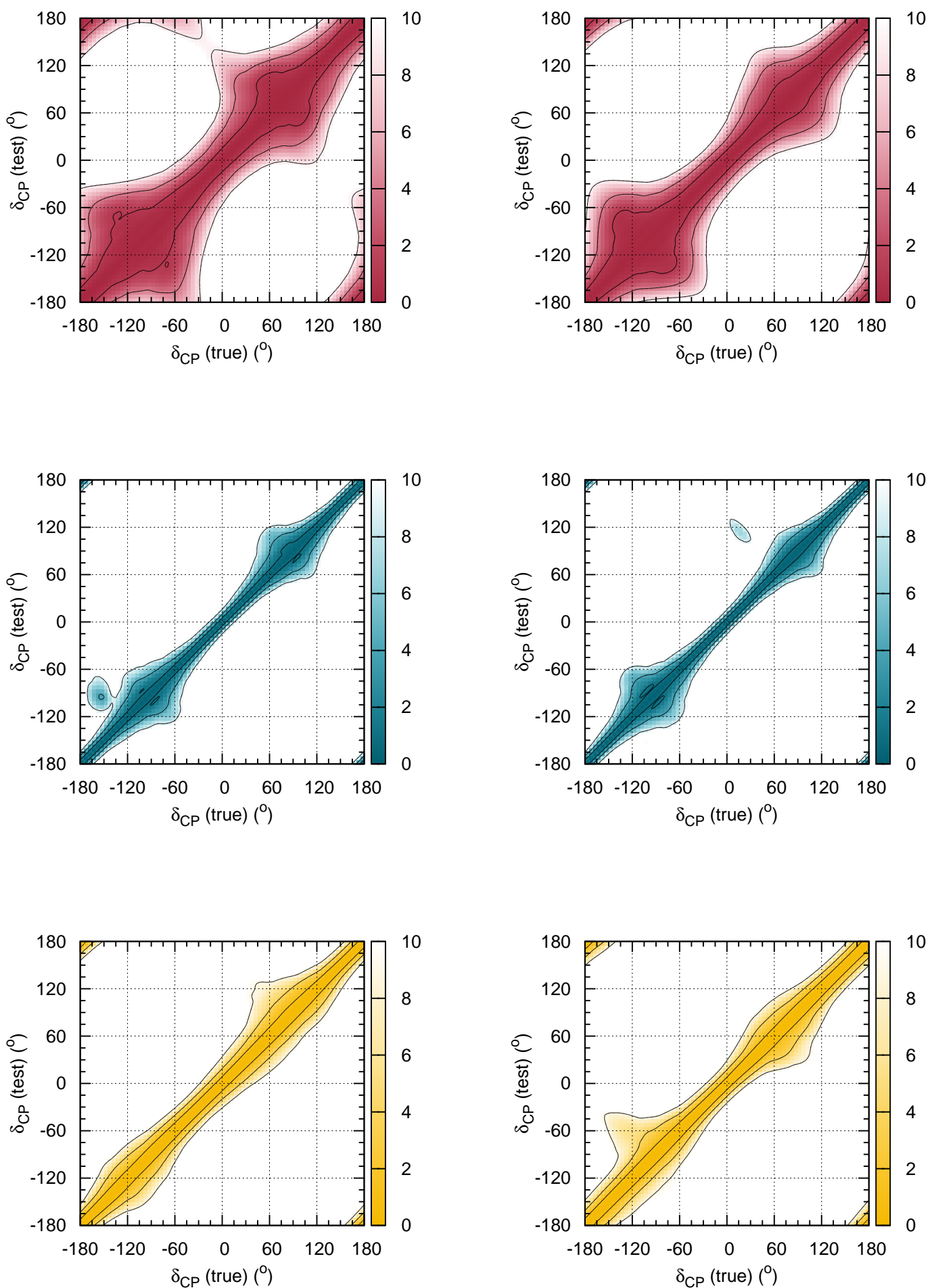

FIG. 2. The allowed range of test $\delta_{\mathrm{CP}}$ for the experiments DUNE (top row), T2HK2 (middle row) and T2Kor (bottom row) as a function of true $\delta_{\mathrm{CP}}$, for true $\theta_{23}=45^{\circ}$ and both true hierarchies - NH (left column) and IH (right column). The coloured shading along the z-axis represent $\Delta \chi^{2}$ values, and the contours correspond to $\sqrt{\Delta \chi^{2}}=1,2,3$. 

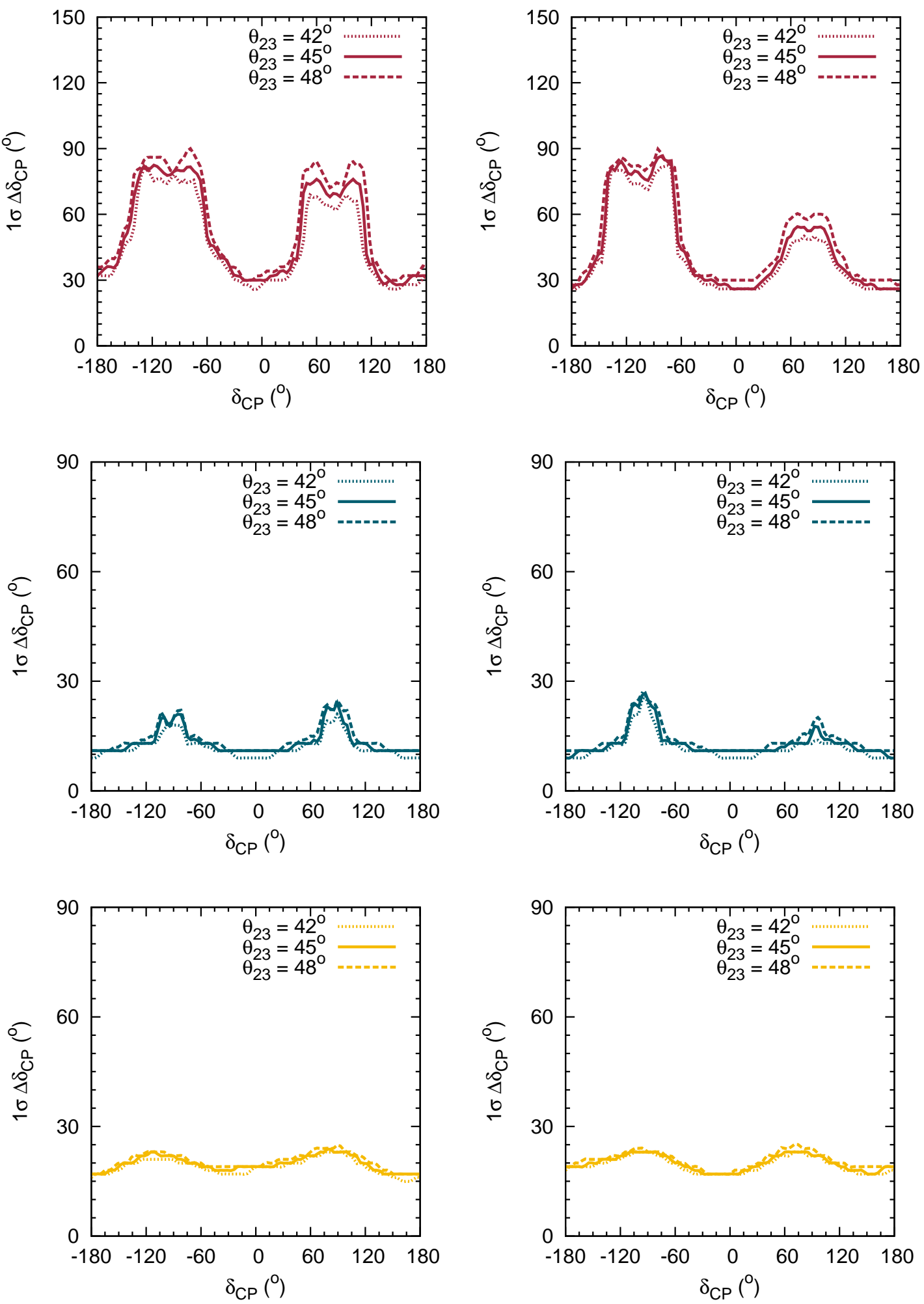

FIG. 3. The $1 \sigma\left(\sqrt{\Delta \chi^{2}}=1\right)$ uncertainty in the allowed $\delta_{\mathrm{CP}}$ range for the experiments DUNE (top row), T2HK2 (middle row) and T2Kor (bottom row) as a function of true $\delta_{\mathrm{CP}}$. The curves correspond to three representative values of $\theta_{23}$ and are shown for both true hierarchies - NH (left column) and IH (right column). The uncertainties are calculated as $360^{\circ} \times$ the fraction of allowed $\delta_{\mathrm{CP}}$ values and correspond to twice the usual error. 
where $\sigma_{\xi}$ is the $1 \sigma$ systematic error corresponding to the experiment in question. In simulating the T2HKK setup, the systematics between the 'constituent experiments' T2HK1 and T2Kor are correlated, unless specified otherwise. In this correlated case, the $\chi^{2}$ is calculated as

$$
\chi^{2}\left(\vec{p}_{\text {true }}, \vec{p}_{\text {test }}\right)=\min _{\xi}\left[\left(\sum_{e \in \operatorname{expt}} \sum_{i \in \text { bins }} \frac{\left(N_{e, i}^{\text {true }}-N_{e, i}^{\text {test }}(\xi)\right)^{2}}{N_{e, i}^{\text {true }}}\right)+\frac{\xi^{2}}{\sigma_{\xi}^{2}}\right] .
$$

In our simulations, we marginalize over the $3 \sigma$ ranges of $\theta_{13}, \theta_{23}, \Delta m_{31}^{2}$ and $\delta_{\mathrm{CP}}$ given by the global fit [13. We include a Gaussian prior on $\sin ^{2} 2 \theta_{13}$ with an error of 0.005 , to account for the reactor neutrino constraint on this parameter. While marginalizing over $\Delta m_{31}^{2}$ and $\theta_{23}$ in discrete steps, we find it useful to include the three-flavour corrections [68 70] in order to avoid spurious contributions to the sensitivity. We do not assume that the mass hierarchy is known, i.e. we allow the test value of $\Delta m_{31}^{2}$ to be both positive and negative and choose the minimum of the two.

\section{A. Typical sensitivity of individual setups}

We first show the hierarchy exclusion sensitivity of DUNE, T2HK2 and T2Kor in Fig. 11 The results are shown as a function of the true value of $\delta_{\mathrm{CP}}$ for both hierarchies and three typical values of true $\theta_{23}$. The sensitivity is represented by the quantity $\sqrt{\Delta \chi^{2}}$. (If the conditions required by Wilks' theorem are satisfied, the computed $\Delta \chi^{2}$ follows the $\chi^{2}$-distribution and $n=\sqrt{\Delta \chi^{2}}$ simply indicates the $n \sigma$ confidence level. However, due to the fact that mass hierarchy is a discrete binary parameter, the relation between $\sqrt{\Delta \chi^{2}}$ and the statistical confidence levels is not trivial. We refer the reader to Refs. $71-73$ for detailed discussions on this matter.) Irrespective of the value of $\delta_{\mathrm{CP}}$ or hierarchy, both DUNE and T2Kor are capable of excluding the wrong hierarchy with $\sqrt{\Delta \chi^{2}}>5$.

As expected based on the discussion in Section III, the hierarchy sensitivity is greater for the favourable combinations of hierarchy and $\delta_{\mathrm{CP}}$. We also see that the hierarchy sensitivity of DUNE is the highest while that of T2HK2 is the lowest, due to matter effects. Finally we observe that hierarchy sensitivity increases with $\theta_{23}$, owing to the leading order term in the oscillation probability.

In Fig. 2, we show the values of test $\delta_{\mathrm{CP}}$ that are consistent with a given value of true $\delta_{\mathrm{CP}}$. The colour coding along the z-axis in these contour plots represents the value of $\Delta \chi^{2}$, while the equi-precision contours have been draw for $\sqrt{\Delta \chi^{2}}=1,2,3$. The plots shown here are for true $\theta_{23}=45^{\circ}$. As expected, the allowed values lie close to the true $\delta_{\mathrm{CP}}=$ test $\delta_{\mathrm{CP}}$ line. Here, we find that T2HK2 has the best precision in measuring $\delta_{\mathrm{CP}}$, followed by T2Kor and finally DUNE because of matter effects. The sensitivity is seen to be best around $\delta_{\mathrm{CP}}=0, \pm 180^{\circ}$ and worst around $\pm 90^{\circ}$. This is because the precision in $\delta_{\mathrm{CP}}$ is proportional to $\mathrm{d} P_{\mu e} / \mathrm{d} \delta_{\mathrm{CP}}$ which goes as cos $\delta_{\mathrm{CP}}$ near the oscillation maximum.

A more intuitive way of seeing these results is to plot the uncertainty in the measurement of $\delta_{\mathrm{CP}}$ (at the $\sqrt{\Delta \chi^{2}}=1$ level) as a function of its true value. This is shown in Fig. 3 . The uncertainty plotted is simply $360^{\circ} \times$ the fraction of allowed $\delta_{\mathrm{CP}}$ values seen in Fig. 2. In most cases this corresponds to twice the 'usual' error - an important point while comparing these results with the collaboration reports Ref. [28, 32]. (Exceptions are when the allowed range is not symmetric around the true value. See for example the middle left panel of Fig. 2 near true $\delta_{\mathrm{CP}}=-160^{\circ}$.) We find that T2HK2 has the best precision in $\delta_{\mathrm{CP}}$ with an uncertainty of $10^{\circ}-15^{\circ}$ for most values of $\theta_{23}$ and $\delta_{\mathrm{CP}}$, except when $\delta_{\mathrm{CP}}$ is close to $\pm 90^{\circ}$. Note that the mass hierarchy is a free parameter in our analysis. The precision in $\delta_{\mathrm{CP}}$ is found to improve if the mass hierarchy is known.

\section{B. Effect of systematics}

The two constituent long-baseline setups of T2HKK - T2HK1 and T2Kor share a common beam source. The detector technology and size, and hence the detector capabilities are also expected to be the same. Therefore, there is a strong correlation between the systematic effects experienced by these two setups. In this subsection, we consider two cases for the combined T2HKK setup - one in which the systematic errors between the two constituents are uncorrelated, and the other where they are correlated. In the uncorrelated case, the total $\chi^{2}$ is simply the sum of the $\chi^{2}$ values from the pull analyses of the individual experiment: The calculation in the correlated case follows the

$¥$ Ref. [50] discusses the effect of uncorrelated systematics in the context of T2HKK. 

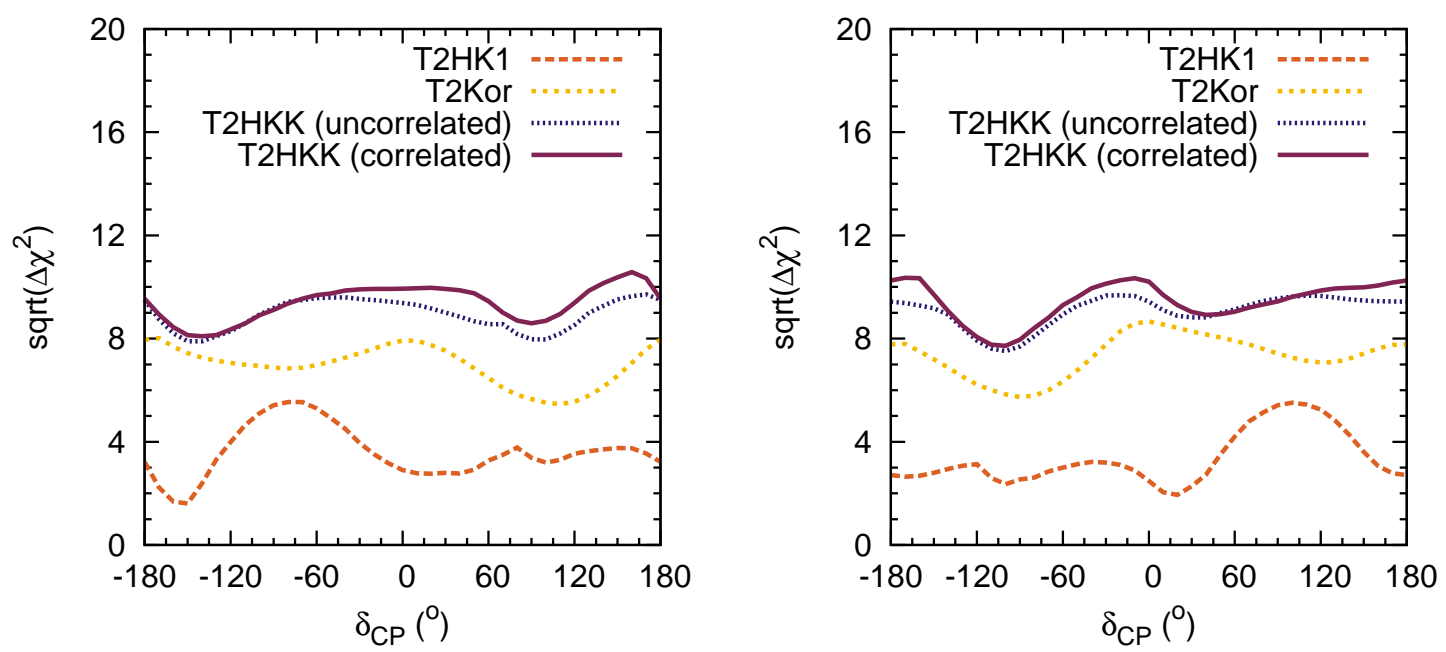

FIG. 4. Hierarchy sensitivity as a function of true $\delta_{\mathrm{CP}}$ for the experiments T2HK1, T2Kor, and their combination T2HKK considering both cases - correlated and uncorrelated systematics. The curves correspond to $\theta_{23}=45^{\circ}$ and are shown for both true hierarchies - NH (left) and IH (right).

method described by Eq. 5. In Fig. 4 we show the result of our computations for the hierarchy exclusion capability. For completeness, we have also showed the hierarchy sensitivity of the individual setups T2HK1 and T2Kor. We find an increase in $\Delta \chi^{2}$ of up to $25 \%$ when the systematic errors are correlated between these experiments. The analysis performed here is very simplistic in its assumptions about the systematic effects, making use of a few effective nuisance parameters. In addition, we assume complete correlation between the systematics at the two constitutent setups. Therefore, our results (for hierarchy sensitivity as well as $\delta_{\mathrm{CP}}$ precision) are slightly more optimistic than the ones in Ref. [32]. Throughout the rest of this study, we will perform our analysis using correlated systematics.

\section{Performance of T2HKK}

Figure 5 shows the performance of the T2HKK setup in excluding the wrong mass hierarchy and in measuring $\delta_{\mathrm{CP}}$ precisely. As the top panel shows, the wrong hierarchy can be excluded at $\sqrt{\Delta \chi^{2}} \gtrsim 7$, irrespective of the hierarchy and values of $\theta_{23}$ and $\delta_{\mathrm{CP}}$. Comparing with Fig. 1, we see that the performance is not as good as DUNE for favourable values of $\delta_{\mathrm{CP}}$ because DUNE has more matter effects. However, it is definitely better than DUNE for unfavourable values of $\delta_{\mathrm{CP}}$ because the shorter baseline data helps to lift the hierarchy- $\delta_{\mathrm{CP}}$ degeneracy by constraining $\delta_{\mathrm{CP}}$. The allowed ranges of $\delta_{\mathrm{CP}}$ seen in the middle row (shown for $\theta_{23}=45^{\circ}$ ) are much smaller than those of the T2Kor setup shown in Fig. 2] thanks to data from the shorter baseline that favours CP-measurement. The $\sqrt{\Delta \chi^{2}}=1$ allowed range is seen to run almost parallel to the diagonal, giving a precision of $10^{\circ}-15^{\circ}$, which is reflected in the lower panel. This corresponds (roughly) to an error of around $5^{\circ}-7.5^{\circ}$ in the measurement of $\delta_{\mathrm{CP}}$.

\section{Comparison of setups}

Finally, we compare the capabilities of the T2HK2 and T2HKK setups in conjunction with DUNE. Since DUNE will anyway collect data at a baseline of $1300 \mathrm{~km}$, these results tell us whether there is any advantage in installing one of the two HK detector tanks in Korea. The top row of Fig. [6 shows the hierarchy discriminating ability of the setups in question as a function of $\delta_{\mathrm{CP}}$, for three representative values of $\theta_{23}$. For all the parameter values under consideration, the wrong hierarchy can be excluded with $\sqrt{\Delta \chi^{2}} \gtrsim 10(13)$ by DUNE+T2HK2 (DUNE+T2HKK). In other words, the combination DUNE+T2HKK outperforms DUNE+T2HK2 by a wide margin. This is to be expected since T2HKK has more matter effects which help in distinguishing the two hierarchies. The middle row shows the allowed $\delta_{\mathrm{CP}}$ regions for the two setups for $\mathrm{NH}$ and $\theta_{23}=45^{\circ}$. Thanks to data from T2HK and the high hierarchy-discriminating ability of the longer baselines, the precision in $\delta_{\mathrm{CP}}$ is high. The uncertainty in $\delta_{\mathrm{CP}}$ is plotted in the bottom row. DUNE+T2HKK has a precision between $10^{\circ}$ and $15^{\circ}$, which is the same precision as T2HKK. On the other hand, DUNE+T2HK2 has better precision close to the CP-conserving values of $\delta_{\mathrm{CP}}=0,180^{\circ}$, which is an improvement 

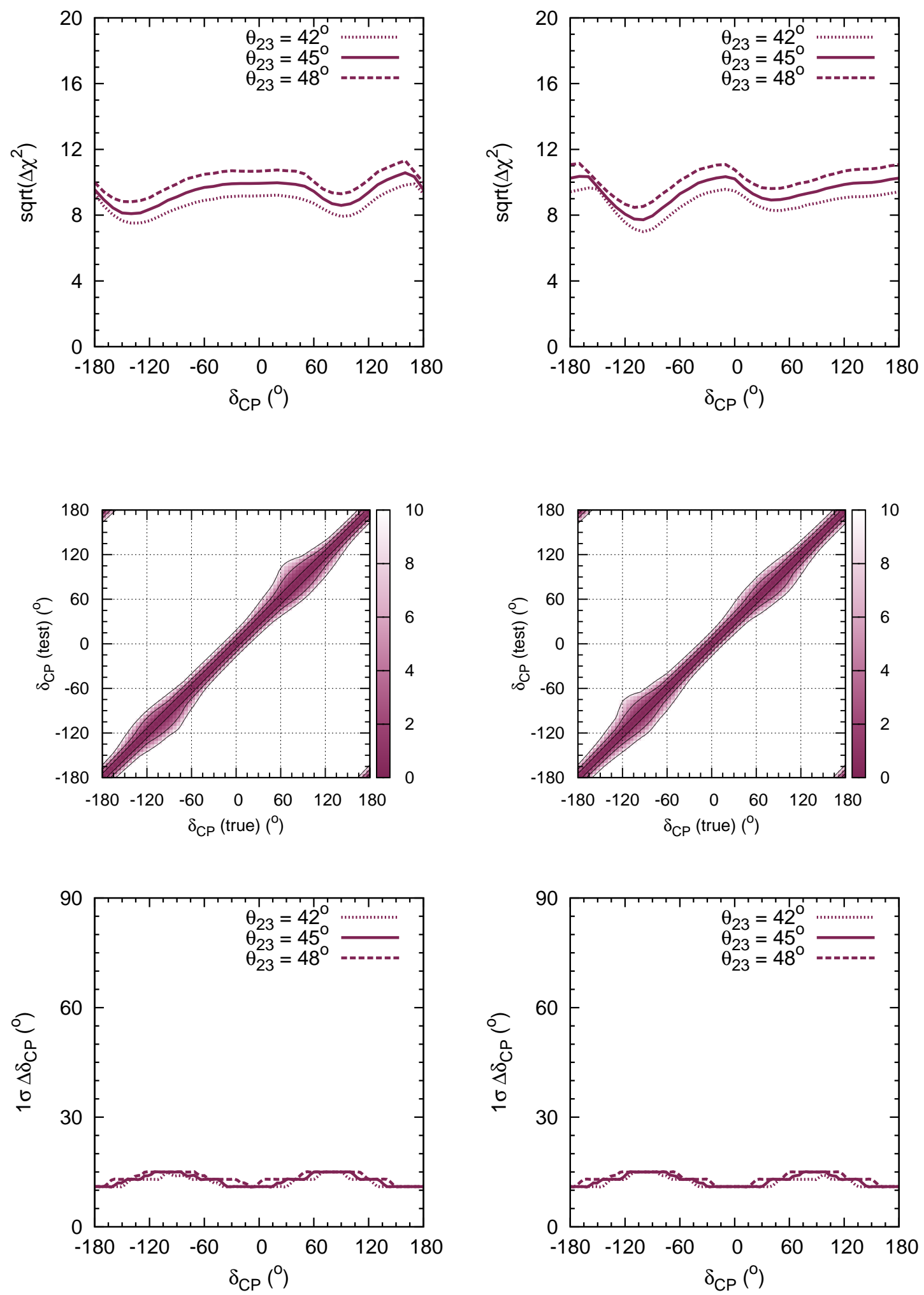

FIG. 5. Top row: Hierarchy sensitivity of T2HKK as a function of true $\delta_{\mathrm{CP}}$. Middle row: The allowed range of test $\delta_{\mathrm{CP}}$ for T2HKK, for $\theta_{23}=45^{\circ}$. Bottom row: The $1 \sigma\left(\sqrt{\Delta \chi^{2}}=1\right)$ uncertainty of the allowed $\delta_{\mathrm{CP}}$ range for T2HKK. All the plots are shown for both true hierarchies - NH (left) and IH (right).

over the precision of T2HK2 alone. Close to the current best-fit value of $-90^{\circ}$, the performance of both setups is 

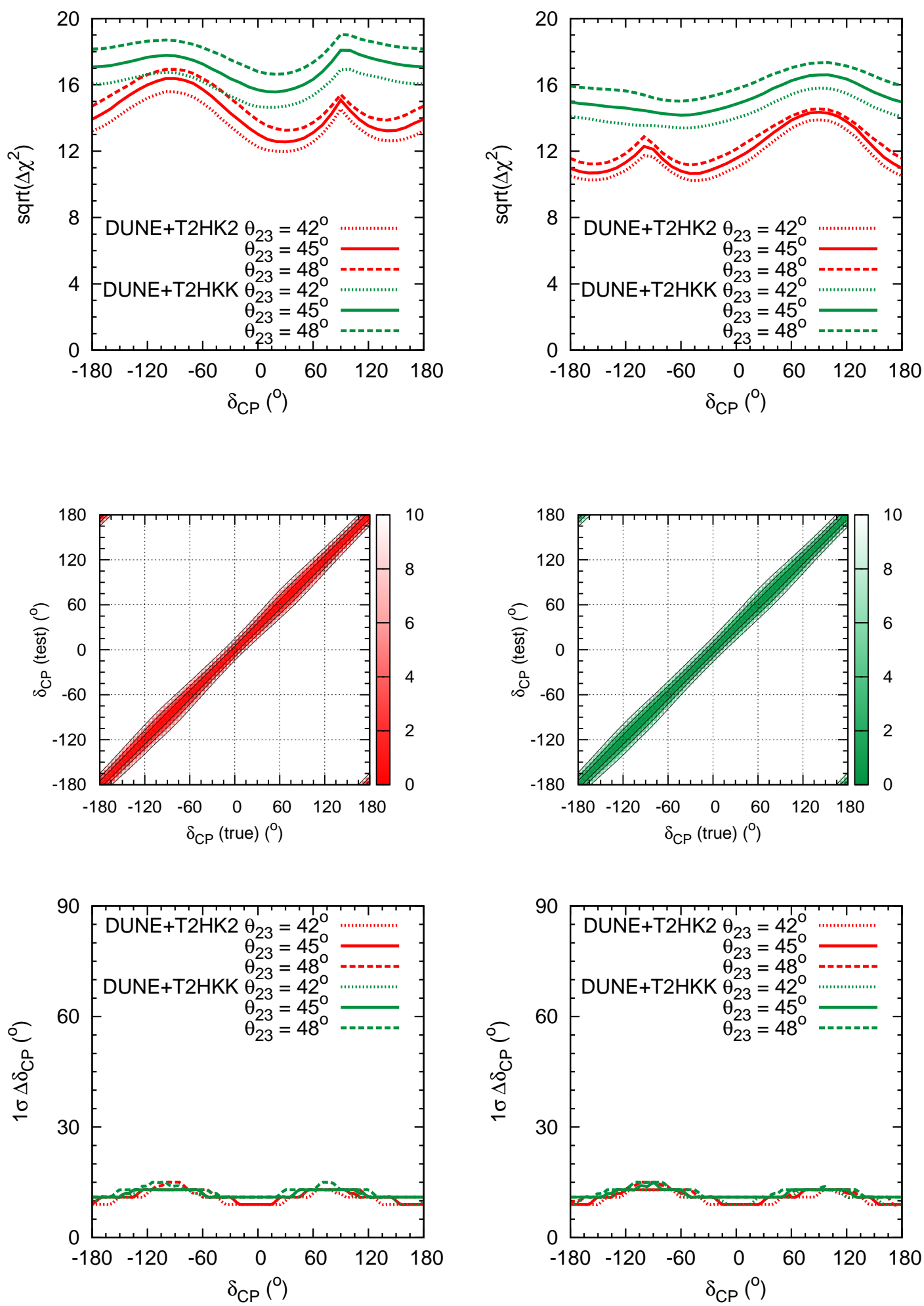

FIG. 6. Physics reach for the combined analysis of DUNE+T2HK2 and DUNE+T2HKK. Top row: Hierarchy sensitivity of DUNE+T2HK2 and DUNE+T2HKK as a function of true $\delta_{\mathrm{CP}}$. The plots are shown for both true hierarchies - NH (left) and IH (right). Middle row: The allowed range of test $\delta_{\mathrm{CP}}$ for DUNE+T2HK2 (left) and DUNE+T2HKK (right), for true $\theta_{23}=45^{\circ}$ and true NH. Bottom row: The $1 \sigma$ $\left(\sqrt{\Delta \chi^{2}}=1\right)$ uncertainty of the allowed $\delta_{\mathrm{CP}}$ range for DUNE+T2HK2 and DUNE+T2HKK. The plots are shown for both true hierarchies $-\mathrm{NH}$ (left) and IH (right). 
comparable.
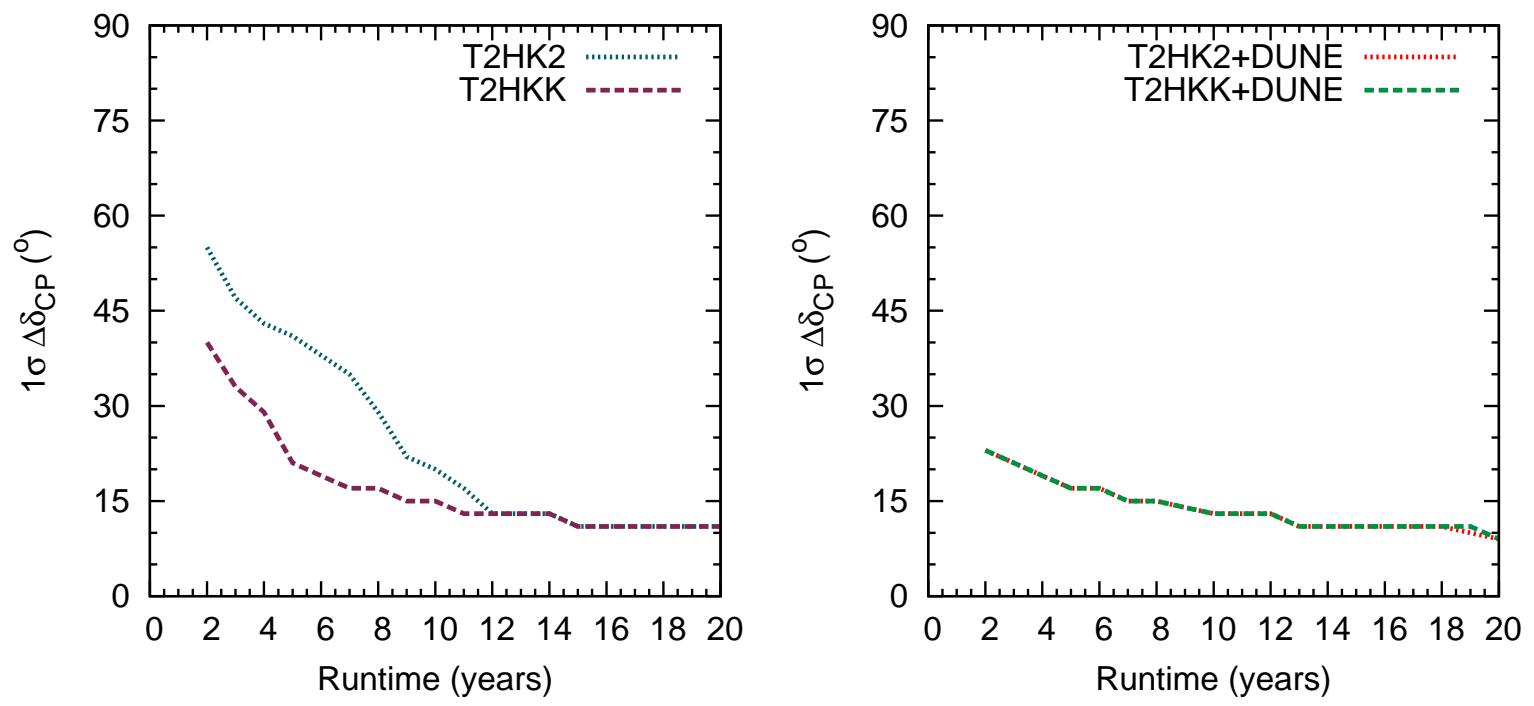

FIG. 7. Left panel: The $1 \sigma\left(\sqrt{\Delta \chi^{2}}=1\right)$ uncertainty in $\delta_{\mathrm{CP}}$ for the experiments T2HK2 and T2HKK as a function of the runtime of the experiment. The curves correspond to $\theta_{23}=45^{\circ}, \delta_{\mathrm{CP}}=-90^{\circ}$ and NH. Right panel: Same as left panel, but in conjunction with full exposure of DUNE.

In Fig. [7, we show the uncertainty in the measurement of $\delta_{\mathrm{CP}}$ with T2HK2 and T2HKK as a function of the runtime of the experiment. The plots have been generated assuming true values $\theta_{23}=45^{\circ}, \delta_{\mathrm{CP}}=-90^{\circ}$ and $\mathrm{NH}$. Here, 10 years corresponds to the benchmark exposure that has been considered throughout this work. Initially as the exposure of the experiment is increased, we see from the left panel that the error in the measurement of $\delta_{\mathrm{CP}}$ is smaller for T2HKK than for T2HK2. This is because T2HKK is capable of breaking the hierarchy- $\delta_{\mathrm{CP}}$ degeneracy because of its long-baseline component. T2HK2 requires around 12 years to achieve the same precision as T2HKK. Further, we see that increasing the runtime beyond 12 years only marginally increases the $\delta_{\mathrm{CP}}$-precision, i.e. there is a saturation of the precision. We have checked that in the ideal case of zero systematics, the $\delta_{\mathrm{CP}}$-precision with T2HK2(T2HKK) can be reduced by around $5^{\circ}\left(2^{\circ}\right)$ over a runtime of 10 years. This emphasizes the role of controlling systematic errors in order to measure $\delta_{\mathrm{CP}}$ with very high precision. With the T2HKK setup, we have a unique configuration with two far detectors in addition to a near detector collecting data using the same source. Improved measurements of the flux and cross-section using this multi-detector setup will allow us to severely constrain the systematic effects, contributing towards a more precise measurement of $\delta_{\mathrm{CP}}$. The curves in the right panel show the corresponding sensitivity after including the full exposure of DUNE. The combined sensitivity is better than the ones in the left panel, as expected. In addition, we see that both combinations perform equally well. Note that the question of which combination is better will depend on the true value of $\delta_{\mathrm{CP}}, \theta_{23}$ and mass hierarchy in nature, as seen from the bottom panels of Fig. 6] The difference between the $\delta_{\mathrm{CP}}$-uncertainty of both combinations is always less than $3^{\circ}$.

\section{SUMMARY AND CONCLUSIONS}

The recent T2HKK proposal envisages placing one of the two detector tanks of HK at a distant site in Korea in the path of the J-PARC neutrino beam. This will allow the observation of neutrinos after propagating for a distance of around $1100 \mathrm{~km}$, in addition to the data being collected by the first detector tank at the original HK site in Japan. The combined setup will be capable of observing neutrino oscillations in the presence (absence) of matter effects through the detector in Korea (Japan).

The measurement of the neutrino mass hierarchy and $\delta_{\mathrm{CP}}$ are two of the outstanding problems in neutrino physics today. Efforts towards measuring them at current facilities are impeded by the hierarchy- $\delta_{\mathrm{CP}}$ degeneracy. This degeneracy can be lifted and the hierarchy can be measured in the presence of substantial matter effects. On the other hand, a precise measurement of $\delta_{\mathrm{CP}}$ requires high statistics and small matter effects. Thus, the T2HKK setup is well suited to simultaneously measure both these unknowns.

Meanwhile, the DUNE experiment will collect neutrino oscillation data with a baseline of $1300 \mathrm{~km}$, with substantial matter effects. Naturally, such a setup has been shown to have very good sensitivity to the mass hierarchy. In light of 
the fact that DUNE will resolve the issue of the mass hierarchy, it is worth investigating whether the second HK tank should be placed in Korea (giving more matter effects and hierarchy sensitivity) or at the HK site itself (giving high statistics with small matter effects, to enhance CP sensitivity). In this work, we have studied the combined capability of DUNE+T2HK2 and DUNE+T2HKK to address this issue.

First, we have presented the capabilities of the individual setups towards determining the mass hierarchy and measuring $\delta_{\mathrm{CP}}$. We find the physics reach of these setups in accordance with our physical understanding of the physics of neutrino oscillations, matter effects and parameter degeneracies. We then combine the T2HK1 and T2Kor setups to test the capability of T2HKK. Through a simplistic treatment of the systematic effects, we show that performing a correlated systematic analysis increases the sensitivity of the experiment by up to $25 \%$. We find that this setup is capable of excluding the wrong hierarchy with $\sqrt{\Delta \chi^{2}}>7$, and measuring $\delta_{\mathrm{CP}}$ with an uncertainty of around $10^{\circ}-15^{\circ}$.

Finally, we conduct a comparison of the setups DUNE+T2HK2 and DUNE+T2HKK. As far as hierarchy discrimination is concerned, DUNE+T2HKK with an exclusion sensitivity of $\sqrt{\Delta \chi^{2}} \gtrsim 13$ outperforms DUNE+T2HK2 purely because of more matter effects. Over a large part of the parameter space including around the current best-fit point, the two setups perform equally well, with a $\delta_{\mathrm{CP}}$ uncertainty of around $10^{\circ}-15^{\circ}$. The precision can be improved by $2^{\circ}-5^{\circ}$ by reducing the systematics (which is possible with a multi-detector setup like T2HKK) and collecting more data. While an improvement from $15^{\circ}$ to $10^{\circ}$ in the precision of $\delta_{\mathrm{CP}}$ may not seem like much, it is significant if $\delta_{\mathrm{CP}}$ truly lies close to the maximally CP-violating value of $-90^{\circ}$ which could signal a new symmetry of nature. In conclusion, both setups offer the possibility of measuring the unknown parameters to very good precision. While the setup with T2HKK is better at determining the mass hierarchy, the setup involving T2HK2 gives the same or better $\delta_{\mathrm{CP}}$ precision, depending on the parameter values chosen. For the current best-fit values, the capabilities of the two setups are comparable.

\section{ACKNOWLEDGEMENTS}

The author would like to thank Monojit Ghosh, Kaoru Hagiwara and Hye-Sung Lee for useful discussions. This work was supported by IBS under the project code IBS-R018-D1.

[1] ATLAS collaboration, G. Aad et al., Observation of a new particle in the search for the Standard Model Higgs boson with the ATLAS detector at the LHC, Phys. Lett. B716 (2012) 1-29, 1207.7214.

[2] CMS collaboration, S. Chatrchyan et al., Observation of a new boson at a mass of 125 GeV with the CMS experiment at the LHC, Phys. Lett. B716 (2012) 30-61, 1207.7235.

[3] SNO collaboration, B. Aharmim et al., Combined Analysis of all Three Phases of Solar Neutrino Data from the Sudbury Neutrino Observatory, 1109.0763

[4] Super-Kamiokande collaboration, R. Wendell et al., Atmospheric neutrino oscillation analysis with sub-leading effects in Super-Kamiokande I, II, and III, Phys.Rev. D81 (2010) 092004, 1002.3471.

[5] KamLAND collaboration, S. Abe et al., Precision Measurement of Neutrino Oscillation Parameters with KamLAND, Phys.Rev.Lett. 100 (2008) 221803, 0801.4589.

[6] T2K collaboration, K. Abe et al., Precise Measurement of the Neutrino Mixing Parameter theta $a_{23}$ from Muon Neutrino Disappearance in an Off-Axis Beam, Phys. Rev. Lett. 112 (2014) 181801, 1403.1532.

[7] NOvA collaboration, P. Adamson et al., First measurement of muon-neutrino disappearance in NOvA, Phys. Rev. D93 (2016) 051104, 1601.05037.

[8] Double Chooz collaboration, Y. Abe et al., Improved measurements of the neutrino mixing angle $\theta_{13}$ with the Double Chooz detector, JHEP 10 (2014) 086, 1406.7763.

[9] Daya Bay collaboration, F. P. An et al., Measurement of electron antineutrino oscillation based on 1230 days of operation of the Daya Bay experiment, 1610.04802 .

[10] RENO collaboration, J. H. Choi et al., Observation of Energy and Baseline Dependent Reactor Antineutrino Disappearance in the RENO Experiment, Phys. Rev. Lett. 116 (2016) 211801, 1511.05849.

[11] G. Fogli, E. Lisi, A. Marrone, D. Montanino, A. Palazzo et al., Global analysis of neutrino masses, mixings and phases: entering the era of leptonic CP violation searches, Phys.Rev. D86 (2012) 013012, 1205.5254.

[12] D. V. Forero, M. Tortola and J. W. F. Valle, Neutrino oscillations refitted, Phys. Rev. D90 (2014) 093006, 1405.7540.

[13] I. Esteban, M. C. Gonzalez-Garcia, M. Maltoni, I. Martinez-Soler and T. Schwetz, Updated fit to three neutrino mixing: exploring the accelerator-reactor complementarity, JHEP 01 (2017) 087, 1611.01514].

[14] T2K collaboration, K. Abe et al., Measurements of neutrino oscillation in appearance and disappearance channels by the T2K experiment with $6.6 \times 10^{20}$ protons on target, Phys. Rev. D91 (2015) 072010, 1502.01550. 
[15] NOvA collaboration, P. Adamson et al., First measurement of electron neutrino appearance in NOvA, Phys. Rev. Lett. 116 (2016) 151806, 1601.05022.

[16] M. Ghosh, S. Goswami and S. K. Raut, Implications of $\delta_{C P}=-90^{\circ}$ towards determining hierarchy and octant at T2K and T2K-II, Mod. Phys. Lett. A32 (2017) 1750034, 1409.5046].

[17] C. H. Albright and M.-C. Chen, Model Predictions for Neutrino Oscillation Parameters, Phys. Rev. D74 (2006) 113006. hep-ph/0608137.

[18] C. Lam, A 2-3 symmetry in neutrino oscillations, Phys.Lett. B507 (2001) 214-218, hep-ph/0104116.

[19] A. S. Joshipura, E. A. Paschos and W. Rodejohann, A Simple connection between neutrino oscillation and leptogenesis, JHEP 0108 (2001) 029, hep-ph/0105175.

[20] T. Endoh, S. Kaneko, S. Kang, T. Morozumi and M. Tanimoto, CP violation in neutrino oscillation and leptogenesis, Phys.Rev.Lett. 89 (2002) 231601, hep-ph/0209020.

[21] T2K collaboration, Y. Itow et al., The JHF-Kamioka neutrino project, hep-ex/0106019

[22] NO $\nu$ A collaboration, D. Ayres et al., NOvA: Proposal to build a 30 kiloton off-axis detector to study $\nu_{\mu} \rightarrow \nu_{e}$ oscillations in the NuMI beamline, hep-ex/0503053.

[23] ICECube collaboration, R. Abbasi et al., The Design and Performance of IceCube DeepCore, Astropart.Phys. 35 (2012) 615-624, 1109.6096.

[24] S. Prakash, S. K. Raut and S. U. Sankar, Getting the Best Out of T2K and NOvA, Phys.Rev. D86 (2012) 033012 1201.6485 .

[25] S. K. Agarwalla, S. Prakash, S. K. Raut and S. U. Sankar, Potential of optimized NOvA for large $\theta_{13} \&$ combined performance with a LArTPC \& T2K, JHEP 1212 (2012) 075, 1208.3644.

[26] S. K. Agarwalla, S. Prakash and S. U. Sankar, Resolving the octant of theta23 with T2K and NOvA, JHEP 07 (2013) 131, 1301.2574.

[27] HyPer-Kamiokande wORKIng Group collaboration, T. Ishida, T2HK: J-PARC upgrade plan for future and beyond T2K, 1311.5287

[28] DUNE collaboration, R. Acciarri et al., Long-Baseline Neutrino Facility (LBNF) and Deep Underground Neutrino Experiment (DUNE), 1512.06148

[29] HyPeR-KAMIOKANDE collaboration, Hyper-Kamiokande Design Report, KEK-PREPRINT-2016-21, ICRR-REPORT-701-2016-1

[30] ICAL collaboration, S. Ahmed et al., Physics Potential of the ICAL detector at the India-based Neutrino Observatory (INO), 1505.07380

[31] D. J. Koskinen, IceCube-DeepCore-PINGU: Fundamental neutrino and dark matter physics at the South Pole, Mod.Phys.Lett. A26 (2011) 2899-2915

[32] Hyper-Kamiokande Proto- collaboration, K. Abe et al., Physics Potentials with the Second Hyper-Kamiokande Detector in Korea, 1611.06118

[33] K. Hagiwara, Physics prospects of future neutrino oscillation experiments in Asia, Nucl. Phys. Proc. Suppl. 137 (2004) 84-103, hep-ph/0410229.

[34] K. Hagiwara, N. Okamura and K.-i. Senda, Physics potential of T2KK: An Extension of the T2K neutrino oscillation experiment with a far detector in Korea, Phys. Rev. D76 (2007) 093002 , hep-ph/0607255.

[35] K. Hagiwara and N. Okamura, Solving the degeneracy of the lepton-flavor mixing angle theta(ATM) by the T2KK two detector neutrino oscillation experiment, JHEP 01 (2008) 022 , hep-ph/0611058].

[36] K. Hagiwara, T. Kiwanami, N. Okamura and K.-i. Senda, Physics potential of neutrino oscillation experiment with a far detector in Oki Island along the T2K baseline, JHEP 06 (2013) 036, 1209.2763.

[37] V. Barger, D. Marfatia and K. Whisnant, Off-axis beams and detector clusters: Resolving neutrino parameter degeneracies, Phys. Rev. D66 (2002) 053007, hep-ph/0206038.

[38] H. Minakata and H. Nunokawa, Exploring neutrino mixing with low-energy superbeams, JHEP 10 (2001) 001 hep-ph/0108085.

[39] J. Burguet-Castell, M. B. Gavela, J. J. Gomez-Cadenas, P. Hernandez and O. Mena, Superbeams plus neutrino factory: The Golden path to leptonic CP violation, Nucl. Phys. B646 (2002) 301-320, hep-ph/0207080.

[40] H. Minakata, H. Nunokawa and S. J. Parke, Parameter degeneracies in neutrino oscillation measurement of leptonic CP and T violation, Phys. Rev. D66 (2002) 093012, hep-ph/0208163.

[41] V. Barger, D. Marfatia and K. Whisnant, How two neutrino superbeam experiments do better than one, Phys. Lett. B560 (2003) 75-86, hep-ph/0210428.

[42] P. Huber, M. Lindner and W. Winter, Synergies between the first generation JHF-SK and NuMI superbeam experiments, Nucl. Phys. B654 (2003) 3-29, hep-ph/0211300.

[43] P. Huber, M. Lindner, T. Schwetz and W. Winter, First hint for CP violation in neutrino oscillations from upcoming superbeam and reactor experiments, JHEP 0911 (2009) 044, 0907.1896.

[44] M. Ghosh, P. Ghoshal, S. Goswami and S. K. Raut, Synergies between neutrino oscillation experiments: an 'adequate' configuration for LBNO, JHEP 03 (2014) 094, 1308.5979.

[45] M. Ghosh, S. Goswami and S. K. Raut, Maximizing the DUNE early physics output with current experiments, Eur. Phys. J. C76 (2016) 114, 1412.1744.

[46] R. B. Patterson, Prospects for Measurement of the Neutrino Mass Hierarchy, Ann. Rev. Nucl. Part. Sci. 65 (2015) 177-192, 1506.07917.

[47] S. Fukasawa, M. Ghosh and O. Yasuda, Complementarity Between Hyperkamiokande and DUNE in Determining Neutrino Oscillation Parameters, 1607.03758 
[48] S. Fukasawa, M. Ghosh and O. Yasuda, Sensitivity of the T2HKK experiment to the non-standard interaction, Phys. Rev. D95 (2017) 055005, 1611.06141.

[49] J. Liao, D. Marfatia and K. Whisnant, Nonstandard neutrino interactions at DUNE, T2HK and T2HKK, JHEP 01 (2017) 071, 1612.01443.

[50] M. Ghosh and O. Yasuda, Effect of systematics in T2HK, T2HKK and DUNE, 1702.06482

[51] R. Gandhi, P. Ghoshal, S. Goswami, P. Mehta, S. U. Sankar et al., Mass Hierarchy Determination via future Atmospheric Neutrino Detectors, Phys.Rev. D76 (2007) 073012, 0707.1723.

[52] V. Barger, R. Gandhi, P. Ghoshal, S. Goswami, D. Marfatia et al., Neutrino mass hierarchy and octant determination with atmospheric neutrinos, Phys.Rev.Lett. 109 (2012) 091801, 1203.6012.

[53] V. Barger, A. Bhattacharya, A. Chatterjee, R. Gandhi, D. Marfatia and M. Masud, Configuring the Long-Baseline Neutrino Experiment, Phys. Rev. D89 (2014) 011302, 1307.2519.

[54] V. Barger, A. Bhattacharya, A. Chatterjee, R. Gandhi, D. Marfatia and M. Masud, Optimal configurations of the Deep Underground Neutrino Experiment, Int. J. Mod. Phys. A31 (2016) 1650020, 1405.1054.

[55] HK collaboration, "https://wiki.hyperk.org/working-groups/wg8-beam-accelerator/fluxfiles."

[56] E. K. Akhmedov, R. Johansson, M. Lindner, T. Ohlsson and T. Schwetz, Series expansions for three flavor neutrino oscillation probabilities in matter, JHEP 0404 (2004) 078, hep-ph/0402175.

[57] P. A. N. Machado, H. Minakata, H. Nunokawa and R. Zukanovich Funchal, What can we learn about the lepton CP phase in the next 10 years?, JHEP 05 (2014) 109, 1307.3248.

[58] M. Ghosh, P. Ghoshal, S. Goswami and S. K. Raut, Evidence for leptonic CP phase from NODA, T2K and ICAL: A chronological progression, Nucl. Phys. B884 (2014) 274-304, 1401.7243.

[59] M. Ghosh, P. Ghoshal, S. Goswami, N. Nath and S. K. Raut, New look at the degeneracies in the neutrino oscillation

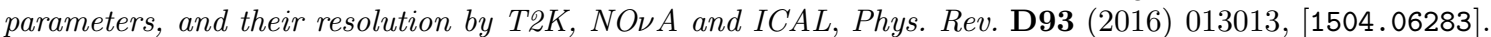

[60] N. Nath, M. Ghosh and S. Goswami, The physics of antineutrinos in DUNE and determination of octant and $\delta_{C P}$, Nucl. Phys. B913 (2016) 381-404, 1511.07496.

[61] P. Huber, M. Lindner and W. Winter, Simulation of long-baseline neutrino oscillation experiments with GLoBES (General Long Baseline Experiment Simulator), Comput.Phys.Commun. 167 (2005) 195, hep-ph/0407333.

[62] P. Huber, J. Kopp, M. Lindner, M. Rolinec and W. Winter, New features in the simulation of neutrino oscillation experiments with GLoBES 3.0: General Long Baseline Experiment Simulator, Comput.Phys.Commun. 177 (2007) 432-438, hep-ph/0701187.

[63] M. D. Messier, Evidence for neutrino mass from observations of atmospheric neutrinos with Super-Kamiokande, UMI-99-23965.

[64] E. Paschos and J. Yu, Neutrino interactions in oscillation experiments, Phys.Rev. D65 (2002) 033002, hep-ph/0107261.

[65] M. Gonzalez-Garcia and M. Maltoni, Atmospheric neutrino oscillations and new physics, Phys.Rev. D70 (2004) 033010. hep-ph/0404085.

[66] G. Fogli, E. Lisi, A. Marrone, D. Montanino and A. Palazzo, Getting the most from the statistical analysis of solar neutrino oscillations, Phys.Rev. D66 (2002) 053010, hep-ph/0206162.

[67] G. Fogli, E. Lisi, A. Marrone and D. Montanino, Status of atmospheric nu(mu) - nu(tau) oscillations and decoherence after the first K2K spectral data, Phys.Rev. D67 (2003) 093006, hep-ph/0303064.

[68] A. de Gouvea, J. Jenkins and B. Kayser, Neutrino mass hierarchy, vacuum oscillations, and vanishing -U(e3)-, Phys.Rev. D71 (2005) 113009, hep-ph/0503079.

[69] H. Nunokawa, S. J. Parke and R. Zukanovich Funchal, Another possible way to determine the neutrino mass hierarchy, Phys.Rev. D72 (2005) 013009, hep-ph/0503283.

[70] S. K. Raut, Effect of non-zero theta(13) on the measurement of theta(23), Mod.Phys.Lett. A28 (2013) 1350093 1209.5658 .

[71] E. Ciuffoli, J. Evslin and X. Zhang, Confidence in a neutrino mass hierarchy determination, JHEP 01 (2014) 095, 1305.5150 .

[72] M. Blennow, P. Coloma, P. Huber and T. Schwetz, Quantifying the sensitivity of oscillation experiments to the neutrino mass ordering, JHEP 03 (2014) 028, 1311.1822.

[73] M. Blennow, On the Bayesian approach to neutrino mass ordering, JHEP 01 (2014) 139, 1311.3183. 